\title{
About the non-standard viewpoint on the dynamics of closed vortex filament
}

\author{
S.V. Talalov \\ Department of Applied Mathematics, State University of Tolyatti, \\ 14 Belorusskaya str., Tolyatti, Samara region, 445020 Russia. \\ svt_19@mail.ru
}

\begin{abstract}
In this article we construct the Hamiltonian description of the closed vortex filament dynamics in terms of non-standard variables, phase space and constraints. The suggested approach makes obvious interpretation of considered system as a quasiparticle that possess certain external and internal degrees of the freedom. The constructed theory is invariant under the transformation of Galilei group. The appearance of this group allows for a new viewpoint on the energy of a closed vortex filament with zero thickness. The explicit formula for the effective mass of the quasiparticle "closed vortex filament" is suggested.
\end{abstract}

keywords: closed vortex filaments; constrained hamiltonian systems; effective mass

\section{Introduction}

The idea that the closed vortex structures have a particle-like properties is very old[1]. Although the development of the fundamental particle physics went in another direction, the dynamics of such structures continues to attract interest [2]. When interpreted as quasiparticles, these structures may possess some unusual properties. For instance, quasiparticle is an object that demonstrates the anizotropic reaction on an external force in some cases. Another example is anyons - the "particles" with fractional statistics. As it seems, such objects can be realized as quasiparticles only.

The investigation of the vortex dynamics in it's different aspects has a long and rich history. Without attempting to review this topic in this short article, let us mention the work [3] where the vortex filament (in the local induction approximation) was discribed in terms of solutions of non-linear Schrödinger equation. It is also relevant to notice here that gauge equivalence between the non-linear Schrödinger equation and the continuous Heisenberg spin chain exists [4]. 
In this work we consider the closed evolving curve $\boldsymbol{z}(\tau, \xi)$ that is defined by the formula

$$
\boldsymbol{z}(\tau, \xi)=\boldsymbol{z}_{0}+R_{0} \int_{0}^{2 \pi}[\xi-\eta] \boldsymbol{j}(\tau, \eta) d \eta
$$

Parameters $\tau$ and $\xi$ are dimensionless parameters. The constant $R_{0}$ is in-put constant in our theory which defines the scale of length. The notation $[x]$ means the integer part of the number $x / 2 \pi$; the $2 \pi$-periodical vector function $\boldsymbol{j}(\xi)$ satisfies the equation of motion

$$
\partial_{\tau} \boldsymbol{j}(\tau, \xi)=\boldsymbol{j}(\tau, \xi) \times \partial_{\xi}^{2} \boldsymbol{j}(\tau, \xi)
$$

and the consraint equalities:

$$
\begin{gathered}
\Phi_{k}=\int_{0}^{2 \pi} j_{k}(\xi) d \xi=0 \quad k=1,2,3, \\
\boldsymbol{j}^{2}(\xi)=1 .
\end{gathered}
$$

Thus, the field $\boldsymbol{j}(\xi)$ defines the $2 \pi$-periodical continuous Heisenberg spin chain[4]. Consequently, the curve $\boldsymbol{z}(\tau, \xi)$ describes closed vortex filament that satisfies the local induction equation:

$$
\partial_{\tau} \boldsymbol{z}(\tau, \xi)=\frac{1}{R_{0}} \partial_{\xi} \boldsymbol{z}(\tau, \xi) \times \partial_{\xi}^{2} \boldsymbol{z}(\tau, \xi)
$$

The space-time symmetry group in our theory is the group $E(3) \times E_{\tau}$, where $E(3)$ is the group of motions of space $E_{3}$ and $E_{\tau}$ is the group of "time translations": $\tau \rightarrow \tau+c$. Let us note that the description of the vortex filament in terms of continuous Heisenberg spin chain still continues to be interesting (see[5], for example).

\section{Dynamical invariants and new variables}

The canonical consideration of the fluid dynamics [6] leads to the following expressions for the momentum and angular momenta:

$$
\boldsymbol{p}=\frac{1}{2} \int \boldsymbol{r} \times \boldsymbol{\omega}(\boldsymbol{r}) d V, \quad \boldsymbol{s}=\frac{1}{3} \int \boldsymbol{r} \times(\boldsymbol{r} \times \boldsymbol{\omega}(\boldsymbol{r})) d V .
$$


The vector $\boldsymbol{\omega}(\boldsymbol{r})$ means the vorticity and the fluid density $\varrho \equiv 1$ here. So, the vorticity for the vortex filament

$$
\boldsymbol{\omega}(\boldsymbol{r})=\Gamma \int_{0}^{2 \pi} \hat{\delta}(\boldsymbol{r}-\boldsymbol{z}(\xi)) \partial_{\xi} \boldsymbol{z}(\xi) d \xi,
$$

where the symbol $\Gamma$ denotes the circulation and the symbol $\hat{\delta}(\xi)$ means $2 \pi$ periodical $\delta$-function. . Thus, for example, the following formula for the canonical momentum takes place:

$$
\boldsymbol{p}=R_{0}{ }^{2} \Gamma \boldsymbol{f}, \quad \boldsymbol{f}=\frac{1}{2} \int_{0}^{2 \pi} \int_{0}[\xi-\eta] \boldsymbol{j}(\xi) \times \boldsymbol{j}(\eta) d \xi d \eta
$$

The similar formula can be written for the angular momenta $\boldsymbol{s}$. The corresponding canonical formula for the energy $\mathcal{E}$ gives the unsatisfactory result because of the divergence of the integral. We will return to this question later.

Now we intend to construct a hamiltonian dynamical system based on the equation (5). In accordance with our suppositions, this equation is the direct consecuence of the representation (11) and the equation (21) for the function $\boldsymbol{j}$. The variable "velocity of liquid" is absent in our theory. Moreover we use the dimensionless "time" $\tau$ in our model. Therefore, the following steps must be done to construct the physically interpreted dynamical system:

1. the formulae for the momentum $\boldsymbol{p}$ and angular momenta $s$ must be added to the equation (2);

2. the value $\Gamma$ is declared an additional dynamical variable in our theory, in relation to variables $\boldsymbol{j}(\xi)$ and $\boldsymbol{z}_{0}$. We denote the set of the dynamical variables $\left\{\boldsymbol{z}_{0}, \Gamma, \boldsymbol{j}(\xi)\right\}$ constrained by the conditions (3) and (4) as $\mathcal{A}$.

3. the additional dimensional constant $t_{0}$ that defines the scale of time, must be added in our theory.

The above considerations lead to the following

Proposition 1 The set $\mathcal{A}$ parametrizes the considered dynamical system the closed vortex filament $\boldsymbol{z}(\xi)$ evolving in accordance with the equation (5) and having a "position" $\left(\boldsymbol{z}_{01}, \boldsymbol{z}_{02}, \boldsymbol{z}_{03}\right)$ and circulation $\Gamma$. This dynamical system has a momentum $\boldsymbol{p}$ and angular momenta $\boldsymbol{s}$ calculated as prescribed above. 
Next we are going to describe the considered dynamical system in terms of other variables. The reasons are following:

- we suppose that new variables will allow to interpret our system clearly as a quasiparticle in a space $E_{3}$;

- we can expand the symmetry group and use the group-theoretical approach for the definition of our systems energy;

- new variables will be more suitable for subsequent quantization 1 .

In fact, we construct the new dynamical system that will be equivalent to the closed vortex filament in some way. To do it let us reparametrize the set $\mathcal{A}$. First, let us note that we can use the equivalent variable $p=|\boldsymbol{p}|$ instead the variable $\Gamma$ on the set $\mathcal{A}$. Second, we introduce the spherical coordinates $(p, \theta, \varphi)$ where the $p_{3}$ axis is parallel to the vector $\boldsymbol{f}$. After that we add the coordinates $(\theta, \varphi)$ as additional dynamical variables. Third, we replace the spherical variables $(p, \theta, \varphi)$ with the Decart variables $p_{1}, p_{2}$ and $p_{3}$ and will use these quantities as new independent fundamental variables. Let us note that corresponding Jakobi determinant $J=p^{2} \sin \theta$ is degenerated on the $p_{3}$ axis. This replacement seems physically justified but we introduce three variables instead of one. To avoid the ambiguity in the reconstruction of the value $\Gamma$ through the new variables $p_{i}(i=1,2,3)$, the new set of dynamical variables must be constrained. Let us consider the constraint

$$
\Phi_{0}\left(p_{1}, p_{2}, p_{1} ; \boldsymbol{j}\right) \equiv(\boldsymbol{p} \boldsymbol{f})^{2}-\boldsymbol{p}^{2} \boldsymbol{f}^{2}=0
$$

Of couse, this equality is fullfilled identically if our theory is parametrized by the set $\mathcal{A}$. We denote the set of the independent variables $\left(\boldsymbol{z}_{0} ; \boldsymbol{p} ; \boldsymbol{j}(\xi)\right)$ constrained by the conditions (3), (4) and (9) as $\Omega$. The following proposition is true.

Proposition 2 The one-to-one correspondence

$$
\mathcal{A} \longleftrightarrow \Omega
$$

is valid.

The proof follows from the Swarz unequality for the vectors $\boldsymbol{p}$ and $\boldsymbol{f}$. One constraint is enough here because the constraint (9) corresponds to the value $\theta=0$ where the Jacobi determinant $J=0$.

\footnotetext{
${ }^{1}$ Quantization is beyond the focus of this article.
} 
The introduction of the variables $p_{i}(i=1,2,3)$ allows to enlarge the space-time symmetry group $E(3) \times E_{\tau}$ of our theory by means of addition of Galilei boosts

$$
p_{j} \longrightarrow \tilde{p}_{j}=p_{j}+c v_{j}, \quad c, v_{j}=\text { const }, \quad j=1,2,3 .
$$

Moreover, we suppose that the procedure of the one-parameter $\left(m_{0}\right)$ central extension for the standard Galilei group $\mathcal{G}_{3}$ is fulfilled. Consequently, the symmetry group for our theory is an extended Galilei group $\widetilde{\mathcal{G}}_{3}$. For convenience, we introduce variables

$$
q_{i}=m_{0} z_{0 i}+\tau t_{0} p_{i}, \quad i=1,2,3
$$

instead of the variables $z_{0 i},(i=1,2,3)$. Finally, the variables $\boldsymbol{j}(\xi), \boldsymbol{q}, \boldsymbol{p}$, will be declared as the new fundamental variables of our model. For example, the curve $\boldsymbol{z}(\tau, \xi)$ must be reconstructed through these variables in accordance with the formula

$$
\boldsymbol{z}(\tau, \xi)=\frac{1}{m_{0}}\left(\boldsymbol{q}-\tau t_{0} \boldsymbol{p}\right)+R_{0} \int_{0}^{2 \pi}[\xi-\eta] \boldsymbol{j}(\tau, \eta) d \eta
$$

\section{$3 \quad$ Energy and hamiltonian structure}

The extension of the symmetry group makes it possible to suggest an energy definition in our model. As it is well-known, the straightforward attempt to calculate the energy of an infinite vortex filament by means of canonical formula [7]

$$
\mathcal{E}=\frac{1}{8 \pi} \iint \frac{\boldsymbol{\omega}(\boldsymbol{r}) \boldsymbol{\omega}\left(\boldsymbol{r}^{\prime}\right)}{\left|\boldsymbol{r}-\boldsymbol{r}^{\prime}\right|} d V d V^{\prime}=\frac{\Gamma^{2}}{8 \pi} \iint \frac{\partial_{\xi} \boldsymbol{z}(\xi) \partial_{\xi} \boldsymbol{z}\left(\xi^{\prime}\right)}{\left|\boldsymbol{z}(\xi)-\boldsymbol{z}\left(\xi^{\prime}\right)\right|} d \xi d \xi^{\prime}
$$

leads to the unsatisfactory result: the integrals in this formula diverge. The standard approach to solve this problem is to take into account the finite thickness $a$ of the filament and the subsequent regularization of the integral. The parameter $a$ is absent in our theory. The energy of the arbitrary configuration in our model will be considered from the group-theoretical viewpoint. Indeed, the Lee algebra of group $\widetilde{\mathcal{G}}_{3}$ has three Cazimir functions:

$$
\hat{C}_{1}=m_{0} \hat{I}, \quad \hat{C}_{2}=\left(\hat{M}_{i}-\sum_{k, j=1}^{3} \epsilon_{i j k} \hat{P}_{j} \hat{B}_{k}\right)^{2}, \quad \hat{C}_{3}=\hat{H}-\left(1 / 2 m_{0}\right) \sum_{i=1}^{3} \hat{P}_{i}^{2}
$$


where $\hat{I}$ is the unit operator, $\hat{M}_{i}, \hat{H}, \hat{P}_{i}$ and $\hat{B}_{i}$ are the respective generators of rotations, time and space translations and Galilean boosts. As it is well known, the function $\hat{C}_{3}$ can be interpreted as an "internal energy of the particle". Let us consider the closed filaments that correspond to the points $p_{i}=0(i=1,2,3), j_{1}(\xi)=\cos \xi, j_{2}(\xi)=\sin \xi, j_{3}(\xi)=0$ (variables $z_{i}$ take arbitrary values) on the set $\Omega$. We postulate here that the energy of this configuration is equal to the value $\mathcal{E}_{0}=m_{0} R_{0}^{2} / t_{0}^{2}$. The constant $\mathcal{E}_{0}$ defines the energy scale in our theory. Regarding the arbitrary configurations, we suppose that the Galilei - invariant expression

$$
\hat{C}_{3}=\frac{\mathcal{E}_{0}}{2 \pi} \int_{0}^{2 \pi}\left(\partial_{\xi} \boldsymbol{j}(\xi)\right)^{2} d \xi
$$

is a natural candidate for the internal energy. Of couse, this expression is motivated by the formula for hamiltonian of continuous Heisenberg spin chain. As a result, the following function on the set $\Omega$ is a good candidate for the energy:

$$
H_{0}\left(p_{1}, p_{2}, p_{3} ; \boldsymbol{j}\right)=\frac{1}{2 m_{0}} \sum_{i=1}^{3} p_{i}^{2}+\frac{\mathcal{E}_{0}}{2 \pi} \int_{0}^{2 \pi}\left(\partial_{\xi} \boldsymbol{j}(\xi)\right)^{2} d \xi .
$$

To complete the consideration of energy, we must define the Poisson brackets that are compatible with the dynamics and constraints. Pursuant to Dirac prescriptions about the primacy of Hamiltonian structure, we define such structure axiomatically here. The correspondent definitions are following.

- Phase space $\mathcal{H}=\mathcal{H}_{j} \times \mathcal{H}_{3}$. The space $\mathcal{H}_{3}$ which is parametrized by the variables $\boldsymbol{q}$ and $\boldsymbol{p}$ is the phase space of a free structureless $3 D$ particle. The space $\mathcal{H}_{j}$ is parametrized by the $2 \pi$-periodical functions $j_{k}(\xi)$, where $k=1,2,3$.

- Poisson structure:

$$
\begin{aligned}
\left\{p_{i}, q_{j}\right\} & =m_{0} \delta_{i j}, \quad i, j=1,2,3 \\
\left\{j_{a}(\xi), j_{b}(\eta)\right\} & =\beta \epsilon_{a b c} j_{c}(\xi) \hat{\delta}(\xi-\eta), \quad \epsilon_{123}=1 .
\end{aligned}
$$

where $\beta=-2 / \mathcal{E}_{0} t_{0}$. All other brackets vanish. In accordance with the definition (13), the function $\boldsymbol{j}^{2}(\eta)$ annulates the brackets of the fundamental variables. Thus the condition (4) selects the symplectic sheet in the phase space $\mathcal{H}_{j}$; 
- Constraints: $\Phi_{k}=0$, where $k=0, \ldots, 3$. The functions $\Phi_{k}$ were defined in the eq. (3) and (9);

- Hamiltonian

$$
H=H_{0}+\sum_{k=0}^{3} l_{k} \Phi_{k}
$$

where the function $H_{0}$ was defined by the formula (12) and the values $l_{k}$ are the Lagrange factors.

Proposition 3 The constraints system (3) and (9) is defined first type constraints in the Dirac terminology.

Indeed, the formulae

$$
\left\{\Phi_{a}, \Phi_{b}\right\}=\beta \epsilon_{a b c} \Phi_{c}, \quad a, b, c=1,2,3
$$

are the direct consequence of the definition (13). Let us calculate the brackets $\left\{\Phi_{a}, \Phi_{0}\right\}$. So, the straightforward calculations lead to the following brackets:

$$
\left\{\Phi_{a}, f_{k}\right\}=-\beta \int_{0}^{2 \pi}[\xi-\eta] j_{k}(\xi) j_{a}(\eta) d \xi d \eta, \quad a, k=1,2,3
$$

that are true if the constraints (3) were are fulfilled. Consequently,

$$
\begin{gathered}
\left\{\Phi_{a}, \Phi_{0}\right\}=2 \beta p^{2} \int_{0}^{2 \pi}[\xi-\eta](\boldsymbol{f} \boldsymbol{j}(\xi)) j_{a}(\eta) d \xi d \eta- \\
-\beta(\boldsymbol{p} \boldsymbol{f}) \int_{0}^{2 \pi}[\xi-\eta](\boldsymbol{p} \boldsymbol{j}(\xi)) j_{a}(\eta) d \xi d \eta-\beta(\boldsymbol{p} \boldsymbol{f}) \int_{0}^{2 \pi}[\xi-\eta](\boldsymbol{p} \boldsymbol{j}(\xi)) j_{a}(\eta) d \xi d \eta .
\end{gathered}
$$

Because the equality $\boldsymbol{p}=$ const $\cdot \boldsymbol{f}$ is fulfilled on the constraint surface (9)), the r.h.s. of this formula is vanished if the equality $\Phi_{0}=0$ holds.

We can easyly prove that the constraint surface $\Omega$ agrees with the dynamics: the equalities $\left\{H, \Phi_{k}\right\}=0$ are fulfilled on the symplectic sheet (4) if the conditions (3) and (9) are fulfilled. This fact means that there are no additional constraints in our theory .

Let us define the "time" $t=t_{0} \tau$. The following equation is true:

$$
\frac{d \boldsymbol{z}(\xi)}{d t}=\frac{\partial \boldsymbol{z}(\xi)}{\partial t}+\left\{H_{0}, \boldsymbol{z}(\xi)\right\}
$$


The calculations of the derivatives here imply that the arguments of the function $\boldsymbol{z}$ are the time $t$ and the fundamental coordinates $\boldsymbol{q}, \boldsymbol{p}, j_{a}(\xi)$ here. The detail calculations with the help of the explicit representation (10) give the final expression for the r.h.s. of the last equality in the form

$$
\left(1 / t_{0} R_{0}\right) \partial_{\xi} \boldsymbol{z}(\xi) \times \partial_{\xi}^{2} \boldsymbol{z}(\xi) .
$$

As a result, we have the following

Proposition 4 The introduced hamiltonian structure defines the hamiltonian flows on the set $\Omega$ correctly. The flow that corresponds to the values $l_{k}=0, k=0, \ldots, 3$, leads to the dynamical equation (5) for the curve $\boldsymbol{z}(\tau, \xi)$ that reconstructed through the coordinates of the space $\mathcal{H}$ in accordance with formula (10).

As usual, we may substitute all constraints explicitly after calculating all the Poisson brackets. Therefore, the natural expression for the energy of our system is:

$$
\mathcal{E}=\left.H\right|_{\Omega}=\frac{1}{2 m_{0}}\left(\boldsymbol{p} \boldsymbol{n}_{f}\right)^{2}+\frac{\mathcal{E}_{0}}{2 \pi} \int_{0}^{2 \pi}\left(\partial_{\xi} \boldsymbol{j}(\xi)\right)^{2} d \xi,
$$

where vector $\boldsymbol{n}_{f}=\boldsymbol{f} /|\boldsymbol{f}|$. This formula leads to the following expression for the inverse effective mass tensor $\left(1 / m_{\mathrm{eff}}\right)_{i k}$ :

$$
\left(\frac{1}{m_{\mathrm{eff}}}\right)_{i k} \equiv \frac{\partial \mathcal{E}}{\partial p_{i} \partial p_{k}}=\frac{1}{m_{0}}\left(\boldsymbol{n}_{f}\right)_{i}\left(\boldsymbol{n}_{f}\right)_{k} .
$$

The model has three dimensional constants: $R_{0}, m_{0}$ and $t_{0}$. These constants define the scale of length, mass and time in our theory.

\section{Concluding remarks}

Among other directions, the important investigations of the closed vortex filament are connected to studying its response to external forces. The experimental studies (see [8, 9], for example) lead to some arguments that this response may be anisotropic. The author hopes that the presented studies have provided further theoretical arguments for such behavior of the vortices. 


\section{References}

[1] W. Thomson, Phil. Mag. 34 (1867) 1524.

[2] K. Moffatt, Rus. J. Nonlin. Dynamics 2 (2006) 401410.

[3] H. Hasimoto, J. Fluid Mech. 23 (1972) 61 - 97.

[4] L.A. Takhtajan, L.D. Faddeev, Hamiltonian Methods in the Theory of Solitons. (Springer-Verlag, Berlin, 1987)

[5] K. Abhinava, P. Guhaby, Inhomogeneous Heisenberg Spin Chain and Quantum Vortex Filament as Non-Holonomically Deformed NLS arXiv: math-ph/1703.02353v2 (2017).

[6] G.K. Batchelor, An Introducton to Fluid Dynamics (Cambrige Univ. Press, 1970).

[7] P.G. Saffman, Vortex dynamics (Cambrige Univ. Press, 1992).

[8] V. F. Kopiev, N. N. Ostrikov. Corona discharge microjets as a possible actuators for jet noise control. TsAGI Science Journal. 41. No1. 97-107 (2010).

[9] V.G. Pimshtein. Direct observation of reaction and scattering of sound on vortices and radiation of sound by vortices in subtonoc turbulent jets at aeroacoustic interaction.TsAGI Science Journal. 43. No 6. $813-820$. (2012). 